\title{
TECNOLOGIAS ENVOLVIDAS NA PROMOÇÃO DA SEGURANÇA DO PACIENTE NO PROCESSO DE MEDICAÇÃO: UMA REVISÃO INTEGRATIVA
}

\author{
Jéssika Wanessa Soares Costa ${ }^{1}$, Jéssica Martinelli Martins de Assis², Marjorie Dantas Medeiros Melo ${ }^{3}$, Suênia \\ Silva de Mesquita Xavierr ${ }^{3}$ Gabriela de Sousa Martins Melo ${ }^{4}$, Isabelle Katherinne Fernandes Costa ${ }^{4}$
}

\begin{abstract}
RESUMO: Este estudo buscou identificar na literatura as tecnologias existentes e seu uso na promoção da segurança do paciente no processo de medicação. Trata-se de uma revisão integrativa realizada nas bases de dados: Literatura Latino-Americana e do Caribe em Ciências da Saúde, Medical Literature Analysisand Retrieval System Online, Índice Bibliográfico Espanhol de Ciências da Saúde, Base de Dados de Enfermagem, PubMed Central, Cumulative Index to Nursing and Allied Health Literature, Web Of Science e SciVerse Scopus, abrangendo artigos publicados entre 2013 e 2017. Vinte artigos internacionais compuseram a amostra final, sendo dez (50\%) publicados no ano de 2014. Onze tecnologias foram identificadas, com prevalência das etapas de prescrição quatro $(36,3 \%)$ e administração de medicamentos quatro (36,3\%), sendo as tecnologias mais abordadas: Radio Frequency Identification, Computerized Physician Order Entry e Bar-Code-Assisted Medication Administration.
\end{abstract}

DESCRITORES: Tecnologia; Sistemas de medicação no hospital; Segurança do paciente; Desenvolvimento tecnológico; Enfermagem.

\section{TECHNOLOGIES INVOLVED IN THE PROMOTION OF PATIENT SAFETY IN THE MEDICATION PROCESS: AN INTEGRATIVE REVIEW}

\begin{abstract}
This study sought to identify technologies existing in the literature and their use in promoting patient safety in the medication process. An integrative review was performed in the following databases: Latin American and Caribbean Health Sciences Literature, Medical Literature Analysis and Retrieval System Online, Spanish Bibliographic Index of Health Sciences, Nursing Database, PubMed Central, Cumulative Index to Nursing and Allied Health Literature, Web of Science and SciVerse Scopus, including articles published between 2013 and 2017. Twenty international articles composed the final sample, ten (50\%) published in the year 2014. Eleven technologies were identified, with prevalence of four regarding prescribed steps (36.3\%) and four regarding drug administration (36.3\%), with the technologies most investigated being: Radio Frequency Identification, Computerized Physician Order Entry and Bar-Code- Assisted Medication Administration.
\end{abstract}

DESCRIPTORS: Technology; Medication systems in the hospital; Patient safety; Technological development; Nursing.

\section{TECNOLOGÍAS ASOCIADAS EN LA PROMOCIÓN DE SEGURIDAD DEL PACIENTE EN EL PROCESO DE MEDICACIÓN: UNA REVISIÓN INTEGRATIVA}

RESUMEN: Este estudio tuvo el propósito de identificar, en la literatura, las tecnologías existentes así como su uso en la promoción de seguridad del paciente en el proceso de medicación. Es una revisión integrativa realizada en las bases de datos: Literatura Latinoamericana y de Caribe en Ciencias de la Salud, Medical Literature Analysisand Retrieval System Online, Índice Bibliográfico Español de Ciencias de la Salud, Base de Datos de Enfermería, PubMed Central, Cumulative Index to Nursing and Allied Health Literature Web Of Science y SciVerse Scopus, abarcando artículos publicados entre 2013 y 2017. Veinte artículos internacionales hicieron parte de la muestra final, siendo diez (50\%) publicados en el año de 2014. Once tecnologías fueron identificadas, con predominancia de las etapas de prescripción, cuatro (36,3\%), y administración de medicamentos, cuatro (36,3\%), siendo las tecnologías más abordadas: Radio Frequency Identification, Computerized Physician Order Entry yBar-Code-Assisted Medication Administration.

DESCRIPTORES: Tecnología; Sistemas de medicaciónen el hospital; Seguridad del paciente; Desarrollo tecnológico; Enfermería.

${ }^{1}$ Enfermeira. Mestranda em Enfermagem. Universidade Federal do Rio Grande do Norte. Natal, RN, Brasil.

${ }^{2}$ Enfermeira. Especialista em Enfermagem do Trabalho. Docente da Universidade Federal do Rio Grande do Norte. Natal, RN, Brasil.

${ }^{3}$ Enfermeira. Doutoranda em Enfermagem. Docente de Enfermagem da Universidade Federal do Rio Grande do Norte. Natal, RN, Brasil.

${ }^{4}$ Enfermeira. Doutora em Enfermagem. Docente de Enfermagem da Universidade Federal do Rio Grande do Norte. Natal, RN, Brasil. 


\section{INTRODUÇÃO}

No transcorrer de todo o processo da prestação da assistência à saúde, os indivíduos tem o direito de receber através dos serviços de saúde uma atenção de qualidade que lhes traga benefícios, assim como satisfação(1).

Os efeitos indesejáveis da assistência à saúde são causados por falhas de qualidade. A qualidade na assistência na prestação do cuidado é entendida como o uso eficiente dos recursos físicos e humanos, com o mínimo de risco ao cliente ${ }^{(2)}$. Dentre as dimensões traçadas pela Organização Mundial de Saúde (OMS), a qualidade dos serviços de saúde divide-se em seis grandes grupos: segurança, efetividade, atenção centrada no paciente, oportunidade/ acesso (interno e externo), eficiência e equidade ${ }^{(3)}$.

Atualmente, a segurança do paciente, uma das dimensões da qualidade, se estabelece como um dos desafios primordiais para garantir a excelência de qualidade no setor de saúde ${ }^{(4)}$, tendo como objetivo a redução do risco de danos desnecessários relacionados aos cuidados de saúde para um mínimo aceitável, de acordo com conhecimento atual, dos recursos disponíveis e no contexto em que os cuidados foram prestados ${ }^{(5)}$.

Ao longo dos anos, houve uma ascensão das preocupações com os eventos adversos ligados às práticas assistenciais. Nesse contexto, os erros de medicação são alvo de estudos, com a finalidade de avaliar e indicar meios para evitar e/ou reduzir os eventos adversos, uma vez que esses erros trazem repercussões negativas e prejuízos para a vida dos indivíduos assistidos. Segundo Joint Commissionon Accreditation of Healthcare Organizations (JCAHO), o processo de medicação possui cinco etapas: seleção e obtenção do medicamento, prescrição, preparo e dispensação, administração de medicamentos e, por último, monitorização do paciente. Estudos demonstram que, ao longo dos anos, foram evidenciados erros durante a execução dessas etapas ${ }^{(6)}$.

O National Reporting and Learning Systems (NRLS) da Inglaterra define um "incidente de segurança do paciente" (PSI) como qualquer acontecimento indesejado ou inesperado. Em estudo realizado, constatou que, entre os anos de 2005 e 2010, mais de 525.186 incidentes foram relatados. Destes, 86.821 $(16 \%)$ casos geraram dano real ao paciente no processo de medicação, e $822(0,9 \%)$ resultaram em morte ou lesão grave ${ }^{(7)}$.

De acordo com a Resolução do COFEN n. 311, de 08 de fevereiro de 2007, os profissionais de enfermagem estão proibidos de executar ações que comprometam a segurança da pessoa, como administrar medicamentos sem conhecer a ação da droga e sem certificar-se da possibilidade de riscos, assim como prescrever medicamentos ${ }^{(8)}$.

Diariamente as novas tecnologias surgem, e os profissionais de saúde necessitam apreendê-las e dominá-las, colocando-as a serviço do cliente, permitindo uma assistência segura e de qualidade ${ }^{(9)}$. As tecnologias na área da saúde são agrupadas em três categorias: duras (material como equipamentos e mobiliário permanente ou de consumo); leve-dura (disciplinas que operam em saúde, a exemplo da clínica médica epidemiológica); e leve (identificada como o processo de produção da comunicação, das relações, de vínculos que conduzem ao encontro do usuário com necessidades de ações de saúde). Todas possuem a mesma finalidade: proporcionar uma assistência ampla e integral ${ }^{(10)}$.

Visando minimizar a ocorrência de eventos adversos no processo de medicação, uma das iniciativas empregadas atualmente é o incentivo do uso das tecnologias com o intuito de subsidiar a prática assistencial, sejam elas eletrônicas ou materiais, bem como fortalecer a relação dos profissionais de saúde com essas tecnologias ${ }^{(11-12)}$. Frente ao exposto, o presente estudo tem como objetivo identificar na literatura as tecnologias existentes para promoção da segurança do paciente no processo de medicação.

\section{- MÉTODO}

Trata-se de revisão integrativa, cuja principal finalidade é construir uma análise da literatura, selecionando e agrupando estudos que envolvem determinada temática, e que tragam características em comum, obtendo aprofundamento e reflexões para subsidiar novos estudos na área ${ }^{(13)}$. 
Para a realização deste estudo, utilizou-se as seguintes etapas: identificação do tema e elaboração da questão de pesquisa, estabelecimento de critérios para inclusão e exclusão de estudos, identificação dos dados a serem obtidos a partir dos estudos selecionados, categorização dos estudos, análise e interpretação dos resultados e apresentação da revisão, com a exposição das etapas percorridas para alcançar os resultados obtidos ${ }^{(14)}$.

Como forma de direcionar esta revisão, formulou-se a seguintes questões de pesquisa: Quais as tecnologias utilizadas no processo de medicação? Quais os impactos do uso das tecnologias na segurança do paciente no processo de medicação?

Durante a busca pelas publicações na literatura cientifica, esta investigação foi realizada no decorrer dos meses de dezembro de 2016 a janeiro de 2017, nas seguintes bases de dados eletrônicas: Biblioteca Virtual em Saúde, Medical Literature Analysis and Retrieval System Online (MEDLINE), Literatura LatinoAmericana e do Caribe em Ciências da Saúde (LILACS), Base de Dados de Enfermagem (BDENF), Índice Bibliográfico Espanhol de Ciências da Saúde (IBECS), PubMed Central, Cumulative Index to Nursing and Allied Health Literature (CINAHL), Web Of Science e SciVerse Scopus (SCOPUS).

Para o levantamento das publicações, utilizou-se os descritores controlados do Medical Subject Headings (MESH): "Medication Systems, Hospital" e "Patient Safety", pesquisados de forma conjunta por meio do operador booleano AND. Toda a etapa de levantamento e seleção dos artigos foi realizada utilizando a metodologia em pares, duas duplas de autores, tendo a avaliação de um terceiro autor na ocorrência de discrepâncias entre os pares, durante a seleção dos artigos.

Para seleção da amostra, os seguintes critérios de inclusão foram adotados: artigos publicados entre 2013-2017 (restrição realizada em decorrência da busca por tecnologias que atualmente estão sendo utilizadas na assistência) e os que apresentassem em seu conteúdo tecnologias utilizadas para assegurar práticas seguras no âmbito do processo de medicação. Foram excluídos os estudos em formato de editorial, carta ao editor, revisões integrativas e sistemáticas, além de artigos duplicados e excluídos durante o processo de determinação da amostra. A Tabela 1 apresenta de forma quantitativa os passos realizados nas bases de dados. Das oito bases de dados analisadas, as bases LILACS, BDENF e IBECS não acrescentaram artigos à amostra final.

Tabela 1 - Estratégia de busca e determinação da amostra seguindo a ordem: Quantitativo dos Estudos Localizados (L), Estudos Relevantes (R), Estudos Duplicados e Excluídos (D), Estudos Lidos na Integra (I) e Estudos Selecionados (S). Natal, RN, Brasil, 2017

CRUZAMENTO

\begin{tabular}{lccccc}
\multicolumn{7}{c}{ CRUZAMENTO } \\
\hline & Medication Systems, Hospital AND Patient Safety & & \\
\hline BASE DE DADOS & $\mathbf{L}$ & $\mathbf{R}$ & $\mathbf{D}$ & $\mathbf{I}$ & $\mathbf{S}$ \\
\hline MEDLINE & 234 & 36 & 11 & 25 & 4 \\
\hline LILACS & 7 & 1 & 0 & 0 & 0 \\
\hline CINAHL & 35 & 14 & 0 & 14 & 1 \\
\hline BDENF & 2 & 0 & 0 & 0 & 0 \\
\hline IBECS & 3 & 0 & 0 & 0 & 0 \\
\hline SCOPUS & 1173 & 35 & 8 & 24 & 7 \\
\hline PUBMED & 5194 & 9 & 2 & 7 & 2 \\
\hline WEB OF SCIENCE & 378 & 48 & 17 & 31 & 6 \\
\hline TOTAL & 7026 & 143 & 38 & 101 & 20
\end{tabular}

Após o procedimento da busca nas bases de dados, as publicações encontradas $(n=7026)$ foram pré-selecionadas com base na leitura do título e resumo. Após a leitura na íntegra das publicações previamente selecionadas $(n=101)$, a amostra final de artigos foi definida $(n=20)$. Em seguida, foram categorizados e analisados de forma descritiva no programa Microsoft Exce ${ }^{\circledR}$ : identificação do artigo (título, autores, bases de dados, local e ano de publicação), objetivos, metodologia do artigo 
(tecnologia abordada e etapa do processo de medicação) e resultados. Foram agrupados de acordo com a classificação de Mehry e colaboradores ${ }^{(11)} \mathrm{em}$ : leve, leve-dura e dura. De maneira seguinte, os resultados dos artigos foram analisados quanto à sua aplicabilidade e efetividade no âmbito da promoção da segurança do paciente no processo de medicação.

\section{RESULTADOS}

Verificou-se que os 20 (100\%) artigos foram publicados no âmbito internacional, sendo dez (50\%) publicados no ano de 2014. Dentre os artigos, 19 (95\%) apresentavam abordagem metodológica quantitativa descritiva. Foram identificadas 11 tecnologias utilizadas no processo de medicação: Radio Frequency Identification (RFID), Bar-Code-Assisted Medication Administration (BCMA), Personal Digital Assistant (PDA), Electronic Medication Administration Record (eMAR), Internet of Things (IoT), Near Field Communication (NFC), Computerized Physician Order Entry (CPOE), Computerized Decision Support Systems (CDSS), HARMLESS; Electronic Prescribing and Decision Support System (ePDSS) e o Healthcare Failure Mode and Effect Analysis (HFMEA). A tecnologia de Radio Frequency Identification (RFID) apresentou-se como a mais citada dentre os artigos pesquisados cinco (25\%).

Houve predomínio das tecnologias nas seguintes etapas: prescrição quatro (36,3\%) e administração quatro $(36,3 \%)$ no processo de medicação. Apenas três $(27,2 \%)$ das tecnologias foram aplicadas em todas as etapas do processo de medicação: HFMEA, IoT e NCF. Dentre a categorização de Menry ${ }^{(11)}$, todas as tecnologias foram classificadas como duras. A Tabela 2 apresenta a distribuição da amostra final dos estudos e as respectivas tecnologias abordadas, assim como a frequência relativa e absoluta, seguidas por sua aplicabilidade.

Tabela 2 - Identificação das tecnologias abordadas pelos artigos selecionados, sua aplicação nas etapas do processo de medicação e categorização tecnológica. Natal, RN, Brasil, 2017

\begin{tabular}{|c|c|c|c|}
\hline Tecnologia & $\begin{array}{c}\text { Amostra } \\
\text { n (\%) }\end{array}$ & $\begin{array}{l}\text { Processo de } \\
\text { Medicação }\end{array}$ & $\begin{array}{c}\text { Categoria } \\
\text { Tecnológica }\end{array}$ \\
\hline Radio Frequency Identification ${ }^{(15-19)}$ & $5(25)$ & Preparo/ADM** & Dura \\
\hline Computerized Physician Order Entry ${ }^{(20-22)}$ & $3(15)$ & Prescrição & Dura \\
\hline Bar-Code-Assisted Medication Administration ${ }^{(23-25)}$ & $3(15)$ & Preparo/ADM** & Dura \\
\hline Electronic Medication Administration Record(26-27) & $2(10)$ & $\mathrm{ADM}^{* *}$ & Dura \\
\hline Healthcare Failure Mode and Effect Analysis ${ }^{(28)}$ & $1(5)$ & $\mathrm{TP}^{*}$ & Dura \\
\hline Computerized Decision Support System ${ }^{(29)}$ & $1(5)$ & Prescrição & Dura \\
\hline HARMLESS ${ }^{(30)}$ & $1(5)$ & Prescrição & Dura \\
\hline Personal Digital Assistant ${ }^{(31)}$ & $1(5)$ & $\mathrm{ADM}^{* *}$ & Dura \\
\hline Internet of Things ${ }^{(32)}$ & $1(5)$ & $\mathrm{TP}^{*}$ & Dura \\
\hline Electronic Prescribing and Decision Support System ${ }^{(33)}$ & $1(5)$ & Prescrição & Dura \\
\hline \multirow[t]{2}{*}{ Near Field Communication $^{(34)}$} & $1(5)$ & $\mathrm{TP}^{*}$ & Dura \\
\hline & $20(100)$ & & \\
\hline
\end{tabular}

*TP: Totalidade do processo.

**ADM: Administração

A tecnologia de Radio Frequency Identification (RFID) permite a captura automática de dados para identificação de objetos providos de dispositivos eletrônicos, hardwares (etiquetas eletrônicas, tags ou transponders) que emitem sinais de radio frequência para leitores que captam estas informações ${ }^{(15-19)}$. O Bar-Code-Assisted Medication Administration (BCMA) ${ }^{(23-25)}$ e Personal Digital Assistant (PDA) ${ }^{(31)}$ são sistemas de leitura de código de barras projetado para evitar erros de medicação na administração, podendo ser registrado pelo Electronic Medication Administration Record (eMAR) ${ }^{(26-27)}$. O Internet 
of Things (IoT) $)^{(32)}$ e o Near Field Communication (NFC) ${ }^{(34)}$ permitem a intercomunicação destas informações.

O Computerized Physician Order Entry (CPOE) é um sistema de prescrição eletrônica que transmite o pedido diretamente para a farmácia, além de apoiar a decisão clínica pela equipe médica ${ }^{(20-22)}$. $\mathrm{O}$ Computerized Decision Support Systems (CDSS) é um software de análise de dados utilizado para preparar um diagnóstico e avaliá-lo(29), ambos podem ser associado ao HARMLES ${ }^{(30)}$, sistema de apoio à decisão clínica, equipado com alertas de erros de prescrição. O Electronic Prescribing and Decision Support System (ePDSS) permite a comunicação entre as informações das prescrições, apoio à decisão clínica, e sistema de alerta para interações entre medicações ${ }^{(33)}$.

O Healthcare Failure Mode and Effect Analysis (HFMEA), uma extensão do Failure Mode and Effect Analysis (FMEA), foi desenvolvido especificamente para cuidados de saúde, atuando na analise de risco e identificação de falhas ${ }^{(28)}$.

O Internet of Things (IoT) ${ }^{(32)}$ e o Near Field Communication (NFC) ${ }^{(34)}$ permitem a intercomunicação de informações e geração de dados por meio de sensores entre equipamentos de leitura.

O Quadro 1 apresenta os resultados obtidos, aplicabilidade da utilização das tecnologias abordadas pelos artigos selecionados neste estudo.

Quadro 1 - Aplicabilidade evidenciada pelos artigos selecionados. Natal, RN, Brasil, 2017

\begin{tabular}{|l|l|}
\hline \multirow{2}{*}{ Tecnologias } & \multicolumn{1}{c|}{ Resultados } \\
\cline { 2 - 2 } & \multicolumn{1}{c|}{ Aplicabilidade das tecnologias } \\
\hline Radio Frequency Identification (RFID) & $\begin{array}{l}\text { Os estudos apontaram o RFID com ferramenta crucial no } \\
\text { processo de identificação dos pacientes, reduzindo os erros } \\
\text { relacionados às trocas de medicações. }\end{array}$ \\
\hline Bar-Code-Assisted Medication Administration & $\begin{array}{l}\text { Estes dispositivos providos de leitores de código de barra não } \\
\text { mostraram grandes divergências positivas entre os setores } \\
\text { hospitalares que utilizam e os que não fazem uso destes } \\
\text { dispositivos. As taxas de redução de erros de medicação foram } \\
\text { mínimas. }\end{array}$ \\
\hline Electronic Medication Administration Record \\
\hline $\begin{array}{l}\text { Healthcare Failure Mode and Effect Analysis } \\
\text { (HFMEA) }\end{array}$ & $\begin{array}{l}\text { O HFMEA mostra-se eficiente na avaliação e detecção precoce } \\
\text { dos possíveis erros que podem atingir determinados processos, } \\
\text { como o de medicação. }\end{array}$ \\
\hline Computerized Decision Support System & $\begin{array}{l}\text { Como suportes atrelados à decisão clínica e à prescrição } \\
\text { médica, estas tecnologias apresentaram resultados positivos } \\
\text { quando utilizadas no ambiente hospitalar. A redução de } \\
\text { alguns fatores foi observada durante a prescrição, como: erros } \\
\text { de ilegibilidade, uso de abreviaturas inadequadas e falta de } \\
\text { informações. }\end{array}$ \\
\hline $\begin{array}{l}\text { Electronic Prescribing and Decision Support } \\
\text { System }\end{array}$ & $\begin{array}{l}\text { Estas tecnologias, envolvidas na intercomunicação de } \\
\text { equipamentos portáteis que realizam a verificação manual de } \\
\text { tags e códigos de barras, possuem integração avançada, além do } \\
\text { baixo custo quando comparadas a outros equipamentos com a } \\
\text { mesma finalidade. São adaptadas para não captar frequências } \\
\text { de outros aparatos presentes no meio hospitalar. }\end{array}$ \\
\hline HARMLESS &
\end{tabular}

As tecnologias, quando introduzidas e testadas no ambiente hospitalar, de modo generalista, apresentaram bons resultados nos processos hospitalares, facilitando a dinâmica de trabalho dos profissionais e/ou reduzindo eventos adversos derivados da assistência prestada, contribuindo para a segurança do paciente. 
O interesse pelos riscos relacionados aos erros de cuidados em saúde cresce a cada dia. Algumas instituições educacionais e hospitalares estão implantando tecnologias e sistemas de monitoramento, com o intuito de preveni-los ${ }^{(32-36)}$. Métodos tradicionais utilizados estão sendo substituídos por tecnologias projetadas para evitar a incidência de erros no processo de medicação ${ }^{(37)}$.

A aplicação das tecnologias de informação (TI) melhoram a qualidade e segurança dos cuidados em saúde, principalmente no processo de medicação ${ }^{(38)}$. O uso de tecnologias duras vêm se intensificando, colaborando para a preocupação de alguns profissionais, seja com o manuseio, a falta de conhecimento ou as falhas no funcionamento que essas tecnologias possam apresentar ${ }^{(39)}$. Estudo analítico descritivo dos relatórios de erros de medicação aponta que as fases mais afetadas pelos erros são a prescrição $(16,4 \%)$ e administração $(71,5 \%)$, em um total de 359 erros de medicação ${ }^{(40)}$, corroborando com os achados desta pesquisa.

A dosagem excessiva é um dos principais equívocos durante a prescrição. A utilização e integração de tecnologias, como a CPOE, podem reduzir em até $20 \%$ a incidência de novos casos de dosagens incorretas, estabelecendo maior margem de segurança para o paciente ${ }^{(20)}$.

Outras tecnologias podem ser agregadas ao processo de prescrição, como o HARMLESS, sistema desenvolvido para apoiar a decisão clínica na etapa de prescrição, alertando para erros de dosagem e interações entre medicamentos prescritos concomitantemente, assim como o ePDSS, que, além destas características, oferece a possibilidade da interligação das informações entre prescrições destinadas a um mesmo paciente ${ }^{(30,33)}$.

Estudos evidenciaram que a aplicação e integração de sistemas, como estes softwares, permitem a redução significativa dos efeitos adversos aos pacientes, reduzindo até mesmo o seu tempo de internação, uma vez que a aplicação do tratamento correto implica em pontos positivos na recuperação, assim como da segurança do cuidado ${ }^{(41)}$.

Pesquisa realizada aponta que o BCMA é eficaz na redução significativa de erros de administração em até $80,7 \%$ e na redução no tempo gasto pela equipe na realização do procedimento. Associado a este software, o eMAR agrega benefícios à etapa de administração, possibilitando que a ação seja documentada em tempo real. Integrada a estas tecnologias, o PDA pode proporcionar mobilidade e praticidade ao procedimento ${ }^{(24)}$.

Erros envolvendo prescrições equivocadas não são alertados pelo BCMA e o eMAR, que operam a partir da correção pelo profissional para que o sistema funcione normalmente ${ }^{(28-29)}$. Estudo realizado em algumas unidades hospitalares, sem a utilização do código de barras eMAR, demonstrou que a taxa de erros de medicação praticamente duplicou (11,5\%) em relação às unidades que faziam uso $(6,8 \%)^{(26)}$.

Diferentemente das tecnologias citadas anteriormente, usadas na administração, a tecnologia RFID apresenta vantagens relacionada à praticidade de uso. Alguma dessas vantagens são a capacidade de verificar sem linha de leitura, vários tags por segundos, maior capacidade no armazenamento de dados e resistência às adversidades do ambiente, superando os dispositivos de código de barras. Facilitando as etapas de preparação e administração de medicamentos, por meio de seus sensores eficientes que não sofrem interferências manuais dos profissionais ${ }^{(42)}$.

Em síntese, esta revisão mostrou que a identificação, com antecedência dos possíveis danos, é fundamental para evitar repercussões negativas à segurança do paciente, com o uso das tecnologias disponíveis.

\section{- CONCLUSÃo}

Esta revisão integrativa identificou, nos artigos selecionados, 14 tecnologias envolvidas na promoção da segurança do paciente no processo de medicação, sendo as mais frequentes a Radio Frequency Identification, Computerized Physician Order Entry e o Bar-Code-Assisted Medication Administration, categorizadas como tecnologias du ras e aplicadas no processo de medicação, no preparo/administração/ 
prescrição e preparo/administração, respectivamente.

As novas tecnologias e sua implementação no âmbito da assistência favorecem a segurança do paciente, de modo a reduzir a ocorrência de erros, principalmente no processo de medicação. Deste modo, esta pesquisa poderá servir como base para orientar, de forma geral, o campo atual das tecnologias de informação aplicadas no cuidado em saúde, interpostos ao processo de medicação.

\section{REFERÊNCIAS}

1. Estepa Del Arbol M, Espadero MCM, Blancas CP, Montero RC. Eficacia de los programas de seguridad del paciente. Enferm Nefrol. [Internet] 2016;19(1) [acesso em 04 jan 2017]. Disponível: http://scielo.isciii.es/pdf/ enefro/v19n1/08_revision1.pdf.

2. Montserrat-Capella D, Cho M, Lima RS. A qualidade do cuidado e a segurança do paciente: histórico e conceitos. In: Agência Nacional de Vigilância Sanitária (ANVISA). Assistência Segura: Uma Reflexão Teórica Aplicada à Prática.Brasília: ANVISA; 2013. p. 13-7.

3. Gama ZAS,Saturno PJ. A segurança do paciente inserida na gestão da qualidade dos serviços de saúde. In: Agência Nacional de Vigilância Sanitária (ANVISA). Assistência Segura: Uma Reflexão Teórica Aplicada à Prática. Brasília: ANVISA; 2013. p. 29-40.

4. Ministério da Saúde (BR). Fundação Oswaldo Cruz; Agência Nacional de Vigilância Sanitária. Documento de referência para o Programa Nacional de Segurança do Paciente. Brasília: Ministério da Saúde; 2014.

5. de Moura GMSS, de Magalhães AMM. A Qualidade do Cuidado e a Segurança do Paciente: histórico e conceitos. In: Agência Nacional de Vigilância Sanitária (ANVISA). Assistência Segura: Uma Reflexão Teórica Aplicada à Prática. Brasília: ANVISA; 2013. p. 65-78.

6. de Magalhães AMM, de Moura GMSS, Pasin SS, Funcke LB, Pardal BM, Krelinget A. Processos de medicação, carga de trabalho e a segurança do paciente em unidades de internação.Rev. esc. enferm. USP. [Internet] 2015;49(n. esp) [acesso em 04 jan 2017]. Disponível: http://dx.doi.org/10.1590/S0080-623420150000700007.

7. NHS England. Patient safety. Stage Three: Directive Improving medication error incident reporting and learning. [Internet] 2014 [acesso em 03 jul 2015]. Disponível: http://www.england.nhs.uk/wp-content/uploads/2014/03/ psa-sup-info-med-error.pdf.

8. Conselho Federal de Enfermagem. Resolução n. 311, de 08 de fevereiro de 2007. Aprova a reformulação do Código de Ética dos profissionais de enfermagem. Rio de Janeiro: COFEN; 2007.

9. de Sabino LMM, Brasil DRM, Caetano JA, Santos MCL, Alves MDS. Uso de tecnología blanda-dura en las prácticas de enfermería: análisis de concepto. Aquichán. [Internet] 2016;16(2 [acesso em 09 jan 2017].Disponível: http://aquichan.unisabana.edu.co/index.php/aquichan/article/view/4310.

10. Merhy EE, Chakkour M, Stéfano E, Santos CM, Rodrigues RA, Oliveira PCP. Em busca de ferramentas analisadoras das tecnologias em saúde: a informação e o dia-a-dia de um serviço, interrogando e gerindo trabalho em saúde. In: Merhy EE, Onocko R, organizadores. Agir em saúde: um desafio para o público. São Paulo: Editora Hucitec; 1997. p.113-50.

11. Abramson EL, Kern LM, Brenner S, Hufstader M, Patel V, Kaushal R. Expert panel evaluation of health information technology effects on adverse events. J. Eval Clin Pract. [Internet] 2014;20(4)[acesso em 25 jul 2015]. Disponível: http://dx.doi.org/10.1111/jep.12139.

12. Cheung KC, van der Veen W, Bouvy ML, Wensing M, van den Bemt PM, de Smet PA. Classification of medication incidents associated with information technology. J. Am Med Inform Assoc. [Internet] 2014;21(e1) [acesso em 25 jul 2015]. Disponível: http://dx.doi.org/10.1136/amiajnl-2013-001818.

13. Teixeira E, Medeiros HP, Nascimento MHM, Costa e Silva BA, Rodrigues C. Integrative literature review stepby-step \& convergences with other methods of review. Rev Enferm UFPI. [Internet] 2014;2(5)[acesso em $17 \mathrm{dez}$ 2016]. Disponível: http://www.ojs.ufpi.br/index.php/reufpi/article/view/1457.

14. Soares LS, Rodrigues IDCV, Martins LN, da Silveira FDR, Figueiredo MLF. Literature review: particularities of 
each type of study. Rev Enferm UFPI. [Internet] 2013;2(n.esp) [acesso em 02 jan 2017]. Disponível: http://www.ojs. ufpi.br/index.php/reufpi/article/view/1200.

15. Chen YY, Tsai ML. An RFID solution for enhancing inpatient medication safety with real-time verifiable grouping-proof. Int J Med Inform. [Internet] 2014;83(1) [acesso em 22 jul 2015]. Disponível:http://dx.doi. org/10.1016/j.ijmedinf.2013.06.002.

16. Kaul SD, Awasthi AK. RFID Authentication Protocol to Enhance Patient Medication Safety. J. Med. Syst. [Internet] 2013;37(6)[acesso em 22 jun 2015]. Disponível: http://dx.doi.org/10.1007/s10916-013-9979-7.

17. Zhang Z1, Qi Q. An efficient RFID authentication protocol to enhance patient medication safety using elliptic curve cryptography. J. Med Syst. [Internet] 2014;38(5)[acesso em 24 jul 2015]. Disponível:http://dx.doi.org/10.1007/ s10916-014-0047-8.

18. Safkhani M, Bagheri N, Naderi M. A note on the security of IS-RFID, an inpatient medication safety. Int. J. Med. Inform. [Internet] 2014;83(1)[acesso em 22 jul 2015]. Disponível:http://dx.doi.org/10.1016/j.ijmedinf.2013.04.003.

19. Jin C, Xu C, Zhang X, Li F. A Secure ECC-based RFID Mutual Authentication Protocol to Enhance Patient Medication Safety. J Med Syst. [Internet] 2016;40(1)[acesso em 22 dez 2016]. Disponível: http://dx.doi.org/10.1007/ s10916-015-0362-8.

20. Cho I, Park H, Choi YJ, Hwang MH, Bates DW. Understanding the nature of medication errors in an ICU with a computerized physician order entry system. PLos ONE. [Internet] 2014;9(12)[acesso em 25 jul 2015]. Disponível: http://dx.doi.org/10.1371/journal.pone.0114243.

21. Zhang Y, Long X, Chen W, Li H, Duan H, Shang Q. A concise drug alerting rule set for Chinese hospitals and its application in computerized physician order entry (CPOE). Springerplus. [Internet] 2016;5(1)[acesso em 22 dez 2016]. Disponível: http://dx.doi.org/10.1186/s40064-016-3701-4.

22. Villamañán E, Larrubia Y, Ruano M, Vélez M, Armada E, Herrero A, et al. Potential medication errors associated with computer prescriber order entry. Int J Clin Pharm. [Internet] 2013;35(4)[acesso em 20 jul 2015]. Disponível: http://dx.doi.org/10.1007/s11096-013-9771-2.

23. Hardmeier A, Tsourounis C, Moore M, Abbott WE, Guglielmo BJ. Pediatric medication administration errors and workflow following implementation of a Bar Code medication administration system. JHealthc Qual. [Internet] 2014;36(4)[acesso em 20 jul 2015]. Disponível:http://dx.doi.org/10.1111/jhq.12071.

24. Bonkowski J, Carnes C, Melucci J, Mirtallo J, Prier B, Reichert E, et al. Effect of barcode-assisted medication administration on emergency department medication errors. AcadEmerg Med. [Internet] 2013;20(8)[acesso em 18 jul 2015]. Disponível:http://dx.doi.org/10.1111/acem.12189.

25. Cochran GL, Barrett RS, Horn DS. Comparison of medication safety systems in critical access hospitals: Combined analysis of two studies. Am J Health Syst Pharm. [Internet] 2016;73(15) [acesso em 22 dez 2016]. Disponível: http://dx.doi.org/10.2146/ajhp150760.

26. Choo J, Johnston L, Manias E. Effectiveness of an electronic inpatient medication record in reducing medication errors in Singapore. Nurs HealthSci. [Internet] 2014;16(2) [acesso em 04 jan 2017]. Disponível: http:/l dx.doi.org/10.1111/nhs.12078.

27. Truitt E, Thompson R, Blazey-Martin D, NiSai D, Salem D. Effect of the Implementation of Barcode Technology and an Electronic Medication Administration Record on Adverse Drug Events. Hospital Pharmacy. [Internet] 2016;51(6)[acesso em 22 dez 2016]. Disponível: http://dx.doi.org/10.1310/hpj5106-474.

28. Khani-Jazani R, Molavi-Taleghani Y, Seyedin H, Vafaee-Najar A, Ebrahimipour H, Pourtaleb A. Risk Assessment of Drug Management Process in Women Surgery Department of Qaem Educational Hospital (QEH) Using HFMEA Method (2013). Iran J Pharm Res. [Internet] 2015;14(2)[acesso em 18 jul 2015]. Disponível: http://www.ncbi.nlm. nih.gov/pmc/articles/PMC4403066/.

29. Zenziper Y, Kurnik DM, Markovits NMD, Ziv A, Shamiss A, Halkin H, et al. Implementation of a clinical decision support system for computerized drug prescription entries in a large tertiary care hospital. Isr Med Assoc J. [Internet] 2014;16(5)[acesso em 20 jul 2015]. Disponível: https://www.ima.org.il/imaj/viewarticle.aspx?ye ar $=2014 \&$ month $=05 \&$ page $=289$. 
30. Lee J, Han H, Ock M, Lee SI, Lee S, Jo MW. Impact of a clinical decision support systemforhigh-alertme dicationsonthepreventionofprescriptionerrors.IntJMedInform. [Internet] 2014;83(12) [acesso em 04 jan 2017]. Disponível: http://dx.doi.org/10.1016/j.ijmedinf.2014.08.006.

31. Ohsaga A, Kondoh K. Bedside Medication Safety Management System Using a PDA and RFID Tags. Medical Information and Communication Technology (ISMICT), 2013 7th International Symposium on. [Internet] 2013 [acesso em 25 jul 2015]. Disponível: http://ieeexplore.ieee.org/stamp/stamp.jsp?arnumber=6521705.

32. Jara AJ, Zamora MA, Skarmeta AF. Drug identification and interaction checker based on IoT to minimize adverse drug reactions and improve drug compliance. Pers Ubiquit Comput. [Internet] 2014;18(1)[acesso em 21 jul 2015]. Disponível: http://dx.doi.org/10.1007/s00779-012-0622-2.

33. Dixon-Woods M, Redwood S, Leslie M, Minion J, Martin GP, Coleman JJ.Improving Quality and Safety of Care Using "Technovigilance": An Ethnographic CaseStudy of Secondary Use of Data from na Electronic Prescribing and Decision Support System. Milbank Q. [Internet] 2013;91(3) [acesso em 28 jul 2015]. Disponível: http://dx.doi. org/10.1111/1468-0009.12021.

34. Özcanhan MH, Dalkılıç G, Utku S. Cryptographically Supported NFC Tags in Medication for Better Inpatient Safety. J Med Syst. [Internet] 2014;38(8)[acesso em 24 jul 2015]. Disponível: http://dx.doi.org/10.1007/s10916-0140061-x.

35. Staff MC. Medication errors: Cut your risk with these tips Medication errors are preventable. Your best defense is asking questions and being informed about the medications you take. Mayo. Clinic. [Internet] 2015 [acesso em 28 jun 2015]. Disponível: http://www.mayoclinic.org/healt.

36. Vaismoradi M, Jordan S, Turunen H, Bondas T. Nursing students' perspectives of the cause of medication errors. NursEduc Today. [Internet] 2014;34(3)[acesso em 27 jul 2015]. Disponível: http://dx.doi.org/10.1016/j. nedt.2013.04.015.

37. Mumcu G, Koksal L, Sisman N, Catar RO, Tarim M. The effect of pharmacy information management system on safety medication use: A study from private hospitals in Istanbul. Marmara Pharmaceutical Journal. [Internet] 2014;18(1)[acesso em 27 jul 2015]. Disponível: http://e-dergi.marmara.edu.tr/marupj/article/view/5000006224.

38. McCullough JS, Casey M, Moscovice I, Prasad S. The effect of health information technology on quality in U.S. hospitals. Health Aff(Millwood). [Internet] 2010;29(4)[acesso em 27 jul 2015]. Disponível: http://dx.doi. org/10.1377/hlthaff.2010.0155.

39. Cargnin MCS, Ottobelli C, Barlem ELD, Cezar-Vaz MR. Tecnologia no cuidado da enfermagem e a carga de trabalho em uti. Rev enferm UFPE on line. [Internet] 2016;10(2)[acesso em 05 dez 2016]. Disponível: http://www. revista.ufpe.br/revistaenfermagem/index.php/revista/article/viewFile/7634/pdf_9771.

40. Redley B1, Botti M. Reported medication errors after introducing an electronic medication management system. J Clin Nurs. [Internet] 2013;22(3-4)[acesso em 26 jul 2015]. Disponível:http://dx.doi.org/10.1111/j.13652702.2012.04326.x.

41. Inchauspe JAF, de Moura JMSS, Carvalho C, da Costa RS. A segurança do paciente no contexto hospitalar: revisão integrativa. Revista de Saúde Dom Alberto.[Internet] 2013;1(1) [acesso em 05 dez 2016]. Disponível: http:// www.lume.ufrgs.br/handle/10183/142288.

42. Ajami S, Carter MW. The advantages and disadvantages of Radio Frequency Identification (RFID) in Healthcare Centers; approach in Emergency Room (ER). Pak J Med Sci. [Internet] 2013;29(Suppl 1)[acesso em 26 jul 2015]. Disponível: http://www.pjms.com.pk/index.php/pjms/article/view/3552/1232. 